\title{
OPeNing the Epithelial Barrier: Osteopontin Preserves Gut Barrier Function During Intestinal Inflammation
}

\author{
Hiroshi Nakase $^{1}$ \\ Published online: 27 October 2018 \\ ○) Springer Science+Business Media, LLC, part of Springer Nature 2018
}

Since the pathophysiology of inflammatory bowel disease (IBD) is multifactorial, a systematic approach to identifying the molecular events that provoke and sustain the chronic, recurring inflammation typical of IBD is required. The continuous stimulation of the mucosal immune system due to the increased permeability of the intestinal epithelial cell (IEC) layer may be the primary defect in patients suffering from IBD, whereas a healthy epithelium provides an effective barrier to the penetration of luminal antigens into the submucosa. Increased paracellular permeability has been documented in the epithelial lining from acutely inflamed and chronically damaged areas of the intestine of IBD patients. Numerous animal studies have demonstrated the development of inflammation in intestinal segments underlying "leaky" mucosa and have even reported increased permeability prior to the onset of intestinal inflammation [1,2].

Intestinal permeability is directly regulated through alteration of the tight junction (TJ)-associated proteins connecting adjacent epithelial cells, or indirectly through their effects on the cytoskeleton. Many inflammatory cytokines regulate $\mathrm{TJs}$ and barrier function by recruiting additional inflammatory cells into the intestinal wall and by internalizing of TJ proteins in IECs and in endothelial cells [3].

Osteopontin (OPN) was identified in 1985 by Heingard et al. as a sialoprotein derived from the bovine bone matrix. OPN was also referred to as secreted phosphoprotein 1 (SPP1) and early T lymphocyte activation-1 (ETA-1) [4], the diversity of its nomenclature reflecting the involvement of OPN in multiple physiological and pathological processes. OPN belongs to the small integrin-binding ligand $\mathrm{N}$-linked glycoprotein (SIBLING) family. Although newly synthesized protein has a molecular mass of approximately $\sim 32 \mathrm{kDa}$, the

Hiroshi Nakase

hiropynakase@gmail.com

1 Department of Gastroenterology and Hepatology, Sapporo Medical University School of Medicine, S-1, W-16, Chuoku, Sapporo, Hokkaido 060-8543, Japan molecular mass of the mature protein is $45-75 \mathrm{kDa}$ due to extensive posttranslational modification. OPN is involved in a variety of biological processes, including bone remodeling, innate immunity, acute and chronic inflammation, and cancer. The expression of OPN is observed in numerous tissues and cells, including intestinal epithelial and immune cells such as macrophages, dendritic cells (DCs), and $\mathrm{T}$ lymphocytes [4]. Importantly, OPN contributes to the efficient development of T helper (Th)-1 immune response and cell survival by inhibiting apoptosis. Several reports of increased concentrations of OPN in the intestinal mucosa and the serum of patients with active IBD suggest that OPN is involved in the development of intestinal inflammation [5]. Experimental studies of the effects of OPN on colonic inflammation, however, have produced conflicting results. In the acute phase of dextran sulfate sodium (DSS)-induced colitis, one study reported worsened colitis in OPN-knockout (KO) mice compared with wild-type mice, with reduced nitric oxide synthase expression and impaired phagocytic activity in intestinal macrophages, whereas another study reported attenuated colitis with reduced macrophage migration in the inflamed colonic tissues of OPN-KO mice. Contrariwise, attenuated colitis was reported in the chronic phase of DSS- and trinitrobenzene sulfonic acid-induced colitis. Toyonaga et al. reported that double OPN/interleukin (IL)10-KO mice had an accelerated onset of colitis compared with single IL-10-KO mice. Nevertheless, OPN-KO mice do not develop spontaneous colitis [5]. Thus, whether OPN ameliorates or exacerbates colonic inflammation is unclear, despite emerging evidence that OPN is associated with the pathophysiology of human IBD. One may question how OPN expression is decreased in intestinal epithelial cells of human IBD and whether, among several molecules, is OPN the most important regulator of occludin phosphorylation.

In this issue of Digestive Diseases and Sciences, Woo et al. provide the new insight into the contribution of OPN to the TJ-associated proteins of intestinal epithelial cells with studies performed in vivo and in vitro [6]. On the basis of 
their data, they concluded that OPN could maintain the TJ complex by enabling the barrier-associated protein occludin to localize to the TJ and subsequently be phosphorylated.

Not surprisingly, significantly intensified disease activity and histological inflammation of OPN-KO mice with DSSinduced colitis were observed in comparison with wild-type mice, confirming previous reports. Woo et al., in further investigations, reported that the expression of occludin in the colonic epithelium was decreased in OPN-KO mice. They also reported the internalization of barrier-forming intercellular occludin to the intracellular compartment, decreased phosphorylation of occludin and decreased transepithelial barrier resistance (TER) in OPN siRNA transfected Caco2 cell lines, whose changes were enhanced by exposure to $5 \%$ DSS.

Although DSS is best known in experimental colitis models, exposure of cultured cells in vitro to DSS was reported to be associated with inflammatory cytokine release and decreased TER [7].

Th1, Th2, and Th17 cytokines are involved in the pathophysiology of ulcerative colitis (UC), whereas Th1 and Th17 cytokines are primarily involved in the pathophysiology of Crohn's disease (CD). Among the many cytokines, tumor necrosis factor (TNF)- $\alpha$ is commonly associated with the pathophysiology of UC and CD. TNF- $\alpha$ induces the transcription factor interferon regulatory factor-1 (IRF-1) in intestinal epithelial cells in vitro, which is associated with epithelial cell apoptosis by OPN suppression [8]. Based on these data, TNF- $\alpha$ could be involved in the destruction of intestinal epithelial cells in human IBD through OPN suppression. Moreover, since IL-13 impairs epithelial barrier function by affecting epithelial apoptosis, TJs, and epithelial restitution, it is likely to be involved in UC pathophysiology [9]. Of note, a recent report documented the contribution of tricellulin, which acts as a "seal" for TJ molecules, via the specific IL-13 receptor $\alpha 2$, toward the regulation of epithelial permeability [10]. Taken together, treatment of targeting the IL-13 signaling pathway is one of the most promising options in the maintenance of intestinal epithelial barrier function in IBD. Therefore, elucidation of the relationship between these cytokine pathways and OPN expression in epithelial cells should yield useful clinical data.

Other than occludin and ZO-1, many other TJ-associated molecules are implicated in regulating paracellular permeability. Although occludin is implicated in the barrier against the passage of macromolecules across mucosa, there are other channel-forming proteins for cations (claudin-2, 10b, 15), for anions (claudin-10a, 17), or for water (claudin-2) in addition to charge-selective barrier-forming claudins (claudin-4, 8, 14) [11]. Most of the junctionassociated proteins can be internalized in response to physiological and pathological stimuli, with subsequent impairment of epithelial barrier function [12]. Unfortunately, Woo et al. only considered the effect of OPN on occludin and ZO-1 and not the many other TJ molecules. Therefore, further investigation will be required to elucidate how OPN affects epithelial barrier function through interaction with other known junctional proteins.

There are the two types of OPN: secreted OPN (sOPN) and an intracellular OPN (iOPN) isoform generated from different OPN translational initiation sites. sOPN is an extracellular protein that relates to numerous physiological
Fig. 1 The contribution of OPN to gut barrier function via occludin

\section{Decreased OPN condition}

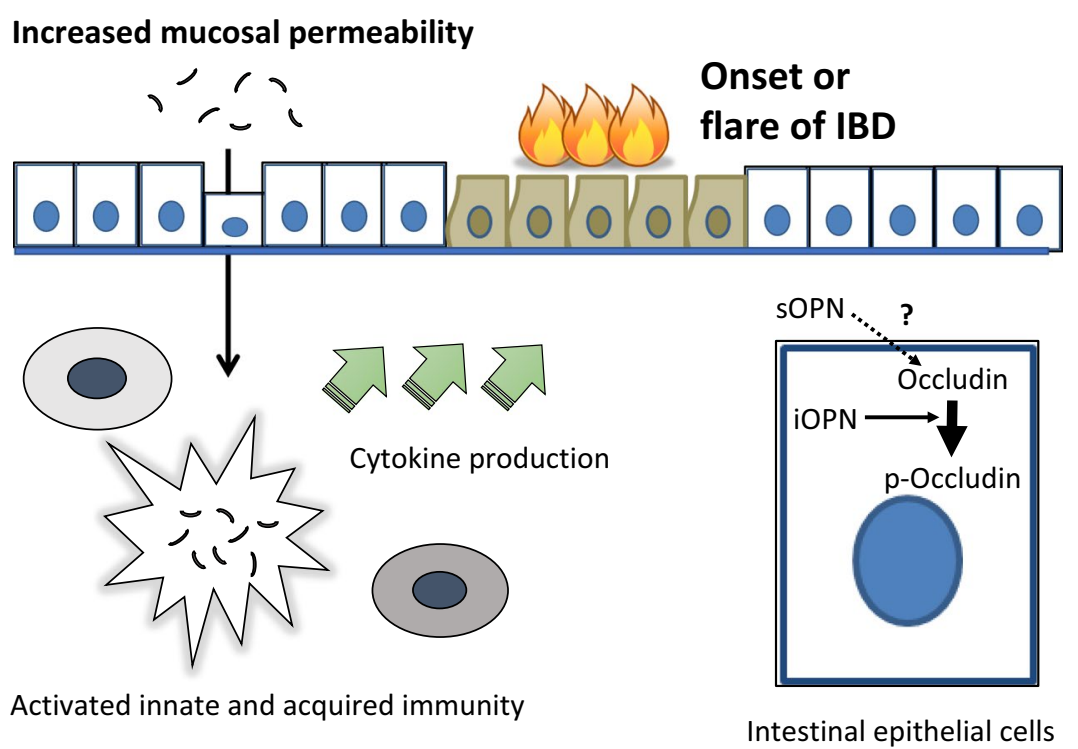


and pathological events. On the contrary, iOPN is predominantly present in the cytoplasm, lacking the $\mathrm{N}$-terminal signal sequence of sOPN [5]. Previous reports primarily demonstrated the effect of OPN administration on increasing the barrier function of $\mathrm{TJ}$-associated molecules via interaction with $\alpha v \beta 3$ integrin and cluster of differentiation (CD44) [4]. These reports did not describe how iOPN may directly contribute to the function of TJ-associated proteins, although in vitro data reported by Woo et al. [6] demonstrated the association between iOPN and occluding expression (Fig. 1). In this regard, Woo's data are intriguing, although the clinical significance of their data remains uncertain.

In summary, dysregulation and internalization of TJassociated proteins are associated with increased intestinal mucosal permeability, which leads to the onset and flare of IBD. The current study provides new data supporting the hypothesis that OPN contributes to the integrity of intestinal mucosa via the prevention of the redistribution and phosphorylation of occludin. Although many issues remain regarding the contribution of OPN to IBD pathophysiology, OPN furtively supports TJ-associated proteins as guardians of the epithelial barrier.

\section{Key Messages}

- Intestinal permeability, which is regulated directly through alteration of tight junction (TJ)-associated proteins, contributes toward the pathophysiology of inflammatory bowel disease.

- The contribution of osteopontin (OPN) toward intestinal inflammation remains unclear.

- OPN maintains the integrity of TJ complex and epithelial barrier integrity during inflammation by preventing the dephosphorylation and internalization of occludin.

\section{References}

1. Bouma G, Strober W. The immunological and genetic basis of inflammatory bowel disease. Nat Rev Immunol. 2003;3:521-533.

2. Madsen KL, Malfair D, Gray D, Doyle JS, Jewell LD, Fedorak $\mathrm{RN}$. Interleukin-10 gene-deficient mice develop a primary intestinal permeability defect in response to enteric microflora. Inflamm Bowel Dis. 1999;5:262-270.

3. Laukoetter MG, Nava P, Nusrat A. Role of the intestinal barrier in inflammatory bowel disease. World J Gastroenterol. 2008;14:401-407.

4. Iida T, Wagatsuma K, Hirayama D, Nakase H. Is osteopontin a friend or foe of cell apoptosis in inflammatory gastrointestinal and liver diseases? Int J Mol Sci. 2018;19:7.

5. Toyonaga T, Nakase H, Ueno S, et al. Osteopontin deficiency accelerates spontaneous colitis in mice with disrupted gut microbiota and macrophage phagocytic activity. PLOS ONE. 2015; 10:e0135552.

6. Woo SH, Lee SH, Park JW, Go DM, Kim DY. Osteopontin protects colonic mucosa from dextran sodium sulfate-induced acute colitis in mice by regulating junctional distribution of occludin. Dig Dis Sci. (Epub ahead of print). doi: https://doi.org/10.1007/ s10620-018-5246-6.

7. Araki $Y$, Sugihara H, Hattori T. In vitro effects of dextran sulfate sodium on a Caco-2 cell line and plausible mechanism for dextran sulfate sodium-induced colitis. Oncol Rep. 2006;16:1357-1362.

8. Tang R, Yang G, Zhang S, Wu C, Chen M. Opposite effects of interferon regulatory factor 1 and osteopontin on the apoptosis of epithelial cells induced by TNF- $\alpha$ in inflammatory bowel disease. Inflamm Bowel Dis. 2014;20:1950-1961.

9. Reinisch W, Panés J, Khurana S, et al. Anrukinzumab, an antiinterleukin 13 monoclonal antibody, in active UC: efficacy and safety from a phase IIa randomized multicentre study. Gut. 2015;64:894-900.

10. Krug SM, Bojarski C, Fromm A, et al. Tricellulin is regulated via interleukin-13-receptor $\alpha 2$, affects macromolecule uptake, and is decreased in ulcerative colitis. Mucosal Immunol. 2018;11:345-356.

11. Krug SM, Schilzke JD, Fromm M. Tight junction, selective permeability, and related diseases. Semin Cell Dev Biol. 2014;36:166-176.

12. Stamatovic SM, Johnson AM, Sladojevic N, Keep RF, Andjelkovic AV. Endocytosis of tight junction proteins and the regulation of degradation and recycling. Ann N Y Acad Sci. 2017;1397:54-65.

\section{Compliance with ethical standards}

Conflict of interest The authors declared that they have no conflict of interest. 